\title{
Efficacy of Laparoscopic Partial Cystectomy with a Transurethral Resectoscope in Patients with Bladder Endometriosis: See-Through Technique
}

\author{
Yuki Endo Jun Akatsuka Kotaro Obayashi Hayato Takeda Tatsuro Hayashi \\ Satoko Nakayama Yasutomo Suzuki Tsutomu Hamasaki Yukihiro Kondo \\ Department of Urology, Nippon Medical School, Tokyo, Japan
}

\section{Keywords}

Bladder endometriosis - Laparoscopic partial cystectomy · Urinary tract endometriosis

\begin{abstract}
Purpose: Bladder endometriosis (BE) is rare. Deep invasive endometriosis is difficult to control with medications alone; such cases need surgical treatment. Good results of laparoscopic partial cystectomy with a transurethral (TU) resectoscope by the see-through technique for patients with BE are reported. Materials and Methods: From January 2008 to February 2016, 12 cases of symptomatic BE were seen in our institution. The chief complaints of 9 cases were micturition pain during menstruation. Preoperative cystoscopy showed a bladder mass with blueberry spots. All surgeries were performed under general anesthesia. Laparoscopic surgery was performed with a fan of 4 ports in the lower abdomen. First, the uterus and bilateral ovaries were checked. Then, the TU resectoscope was inserted. When the affected bladder wall was identified, it was again observed with the laparoscopic light source off, which made it possible to observe the twilight leaking inside from the bladder. This twilight came from the light source of the TU resectoscope via the unaffected bladder wall. In contrast, the thickness of the affected wall prevented the light from inside the bladder from passing through it. We call this the "see-through technique." The tu-
\end{abstract}

\begin{tabular}{ll}
\hline karger@karger.com & (c) 2020 The Author(s) \\
www.karger.com/uin & Published by S. Karger AG, Basel Openger \\
& This article is licensed under the Creative Commons Attribution- \\
Karger & NonCommercial-NoDerivatives 4.0 International License (CC BY- \\
NC-ND) (http://www.karger.com/Services/OpenAccessLicense). \\
Usage and distribution for commercial purposes as well as any dis- \\
tribution of modified material requires written permission.
\end{tabular}

mor was then safely dissected with both laparoscopic and TU resection procedures. Finally, the bladder was sutured by laparoscopic procedures using absorbable sterile surgical suture. The urethral catheter was removed after cystography 7 days after the operation. Results: The surgical margins of all cases were negative. There has been no recurrence of BE so far in any patients. There were no major adverse events perioperatively and the urinary symptoms improved in all cases. Conclusions: By laparoscopic partial cystectomy assisted with a TU resectoscope and see-through technique, the edge of BE could be easily and precisely identified. These procedures are effective and safe for BE surgical treatment.

(C) 2020 The Author(s)

Published by S. Karger AG, Basel

\section{Introduction}

Endometriosis, the presence of endometrial tissue outside the uterine cavity, affects $5-15 \%$ of premenopausal women [1]. The most common sites affected by endometriosis in the pelvic cavity are the torus uterinus, the posterior fornix, the uterosacral ligaments, the rectum, the vagina, and the urinary tract [2]. Urinary tract endometriosis (UTE) is a rare entity that occurs in about $1 \%$ of all endometriotic lesions [3], and the most commonly affected sites are the bladder (85\%), ureter (9\%), kidneys (4\%), and urethra (2\%). The pathogenesis of UTE has not been 
clearly explained. In bladder endometriosis (BE), endometrial lesions affect mainly the detrusor muscle in the bladder trigone and bladder dome [2]. Symptoms depend on the location and size of the changes with menstruation [4]. One-third of patients remain asymptomatic, and the lesions do not exceed 1-2 cm. In symptomatic patients, the main complaints are dysuria, micturition pain, urinary urgency and/or frequency, a burning sensation in the urethra, and discomfort in the lower abdomen. These symptoms are recurrent in $40 \%$ of patients, usually occur in the pre-menstruation period, and are often confused with symptoms of urinary tract infections. Hematuria, which is widely regarded as the dominant symptom of UTE, in fact affects only $20-30 \%$ of patients. This is due to the fact that endometriosis rarely infiltrates deep enough to cause ulceration of the urothelial mucosa [5]. Magnetic resonance imaging (MRI) is considered to be the "gold standard" in UTE diagnosis [6].

There are no substantial guidelines for the treatment of UTE due to the rarity of the disease and the inability to conduct statistical analyses. Treatment of UTE is based on many factors, such as the age of patients, extent of the disease, severity of urinary tract symptoms, and the presence of other foci of endometriosis in the abdomen. In some cases, fertility preferences should be taken into consideration. BE could be treated with drugs, surgery, and combinations of both methods. BE is usually treated with gonadotropin-releasing hormone $(\mathrm{GnRH})$ analogues, gestagens, and combined oral contraceptives. However, almost $50 \%$ of patients with UTE cannot achieve a complete cure with these treatments [7]. What is worse, side effects are frequent with medications, and the medications could be another reason for infertility.

Surgery is the only treatment that gives a chance for a complete cure while maintaining fertility. Patients with $\mathrm{BE}$ are typically young and often desire maintaining an aesthetic appearance. Endometriotic cells infiltrate the bladder from outside toward the urothelial mucosa. While BE cannot be completely dissected by transurethral (TU) resection, laparoscopic partial cystectomy (LPC) has usually been performed.

Seracchioli et al. [8] reported LPC assisted with cystoscopy for BE as a light-to-light technique in 2002. With this technique, the true incision line is still hard to decide for less experienced surgeons. In the present paper, our new "see-through technique" is described. With this fine procedure, the precise edge of the infiltrated lesion can be accurately identified, and the good results with this approach are reported below.

Laparoscopic Partial Cystectomy for

Bladder Endometriosis

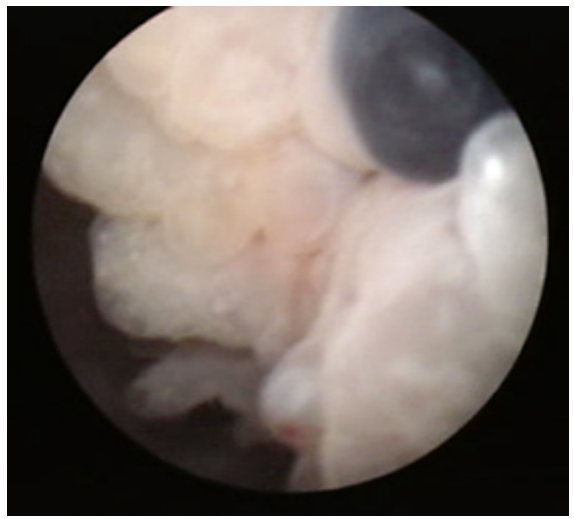

Fig. 1. Blueberry spots on cystoscopy.

\section{Materials and Method}

From January 2008 to February 2016, 12 cases of symptomatic $\mathrm{BE}$ were treated. They included patients with a past history of endometriosis, hormonal therapy for infertility, and hormonal therapy for BE. Preoperative cystoscopy showed a bladder mass with blueberry spots in each case (Fig. 1), and fat-suppressed T1weighted MRI showed a high intensity area in the same place.

All patients underwent partial cystectomy under general anesthesia and in a horizontal supine position, with the lower limbs spread out for the TU resection procedure. Laparoscopic surgery was performed with a fan of 4 ports in the lower abdomen. First, the whole abdominal cavity was checked by gynecologists. Adhesiolysis, ovarian cystectomy, and excision of other pelvic endometriotic nodules, if present, were all performed before urinary surgery. Then, the TU resectoscope was inserted inside the bladder, and the bladder was observed from outside by laparoscopy and inside by the TU resectoscope. When the affected bladder wall was identified, the TU resectoscope was placed in front of the affected wall to illuminate vertically. After turning off the laparoscopic light source, the affected wall was again observed with the laparoscope. By turning off the laparoscopic light source, the whole laparoscopic image became dark. In this darkness, it was possible to observe the twilight leaking inside from the bladder. This twilight came from the light source of the TU resectoscope via the unaffected bladder wall. The unaffected bladder wall was so thin that the light from inside the bladder passed through it. In contrast, the thickness of the affected wall prevented the light from inside the bladder from passing through it. With this phenomenon, it was possible to identify the edge of the affected wall easily and precisely. We call this the "see-through technique" (STT) (Fig. 2-4). Then, a line was placed between the twilight area and the shadow area with margins of $5 \mathrm{~mm}$ using a scissor scale. Next, by TU resectoscope, we put markings around affected urothelial mucosa with margins of $5 \mathrm{~mm}$ using TU cautery knife. The affected wall was then safely dissected with both the laparoscope and the TU resectoscope, keeping away from bilateral orifices observed by the TU resectoscope. Finally, the opened bladder was sutured by laparoscopic procedures using absorbable sterile surgical thread. Cystography was routinely performed on the 7 th day after the operation, and then the urethral catheter was removed. 
Fig. 2. The light-on laparoscopy image showing forceps pulling up the top of the endometriosis lesion.

Fig. 3. The light-off laparoscopic image. This is exactly the same image as shown in Figure 2 but with the laparoscopic light source turned off.

Fig. 4. Sagittal graphic scheme of seethrough technique. The light inside the bladder can pass through the normal bladder wall. The edge of the infiltrated lesion is precisely identified by its shade made by the TU resection light source lit inside the bladder (see-through technique).
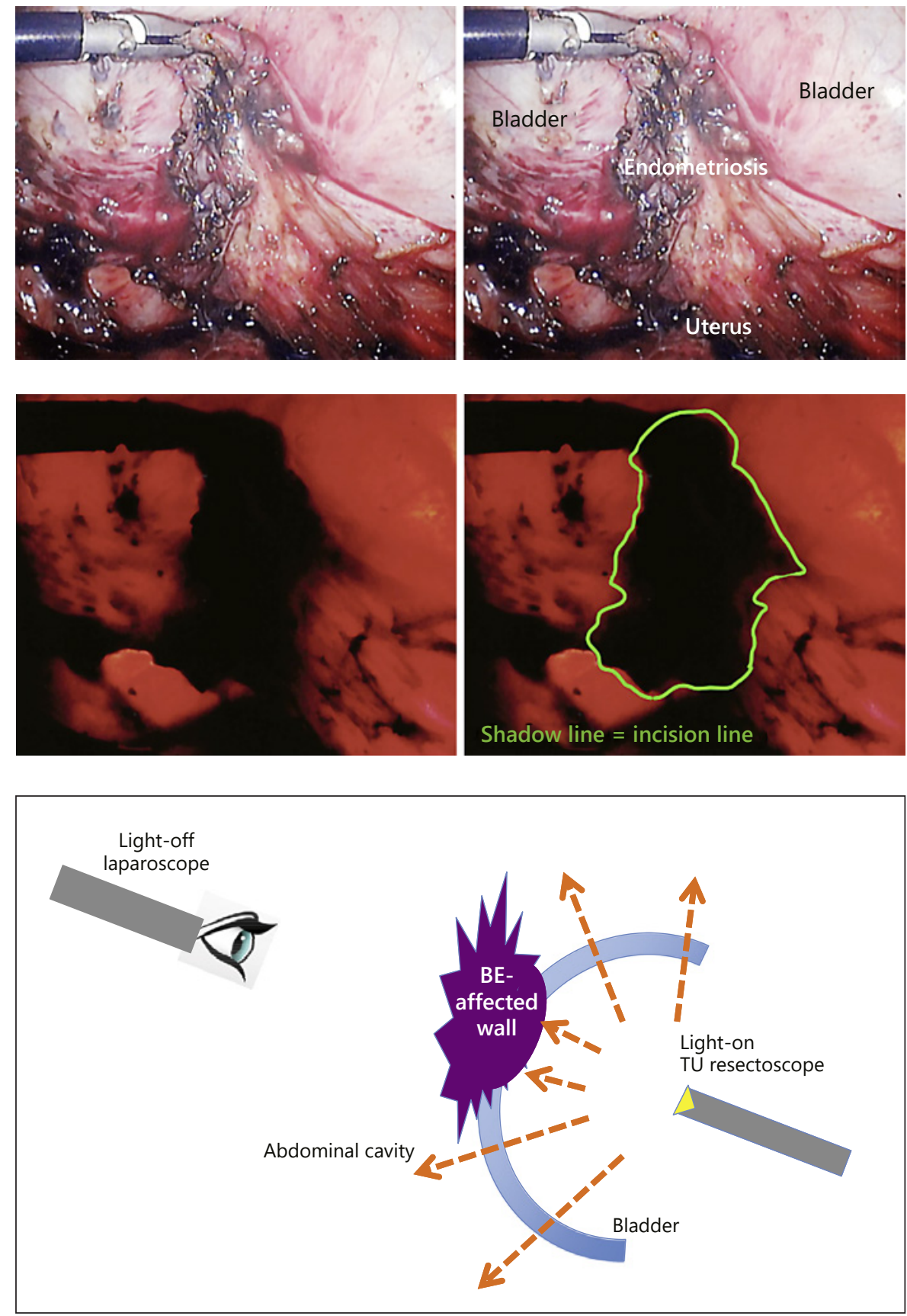

\section{Results}

The background characteristics of all 12 women are shown in Table 1. Their mean age was 41 (range 36-47) years, and $25 \%(3 / 12)$ had a history of endometriosis. Previous hormonal therapy for $\mathrm{BE}$ was performed in $33 \%$ $(4 / 12)$, and $42 \%(5 / 12)$ of the patients complained of infertility. All patients underwent surgical treatment. No in- traoperative complications occurred. The mean operation time was 185 (100-264) min, TU resection time was 47 (22-90) $\mathrm{min}$, and estimated blood loss was $60(0-250) \mathrm{mL}$. There were no adverse events of CTCAE grade III or higher. All symptoms were improved, and all patients indicated they were satisfied. The median follow-up time was 20 (8.6-52.4) months. No patients have had recurrence so far, and two became pregnant after the operation. 
Table 1. Background characteristics of the 12 women with symptomatic bladder endometriosis

Age, years

Operation time, h:min

TUR time, h:min

Blood loss during operation, $\mathrm{mL}$

Hospital stay, days

Follow-up, months

History of endometriosis, \%

Previous hormone therapy, \%

History of surgery, \%

Values are presented as median (range) or rate $(n / N)$. TUR, transurethral resection.

\section{Discussion}

In this study, LPC was performed with a TU resectoscope for BE and resulted in complete cure in all patients. With STT, it was possible to identify the edge of the affected wall correctly and ensure the true incision line. During the average follow-up of 20.4 months, there was no recurrence, and two patients became pregnant.

There are some surgical options available for the patients with BE. TU resection is one surgical approach for BE. Garry [9] reported the combination therapy of TU resection and simultaneous use of $\mathrm{GnRH}$ analogues. However, the relapse rates in such cases are approximately 25 $35 \%$, and there are high rates of vesical perforation $[9,10]$. Since endometriosis originates outside the bladder and invades toward the urothelial mucosa [11], TU resection alone as surgical therapy for $\mathrm{BE}$ is not appropriate.

There are some reports of partial cystectomy for BE [12], with conventional partial cystectomy performed by an open, laparoscopic, or robotic-assisted procedure. They achieved symptom remission rates of $95-100 \%$ and low rates of recurrence regardless of the surgical approach $[12,13]$.

We added the TU resectoscope to LPC, which has three advantages. The first advantage is related to the surgical view. The simultaneous operation of the TU resectoscope with LPC may guide the surgeon in terms of identification of the lesion by laparoscopy and identification of orifices by the TU resectoscope. There is no doubt that, with a better view, surgeons can perform a far more precise and safer operation. Besides, when the affected lesion was large and/or close to an orifice and/or located in the proximal distal bladder wall, the TU resectoscope made it easy to dissect it and stay far from the orifice. The TU resectoscope could provide surgeons with much more safety than

Laparoscopic Partial Cystectomy for

Bladder Endometriosis
LPC. The second advantage is the ability to perform STT. When the laparoscopic light source is turned off during observation, STT can be performed instantaneously. The TU resectoscope is essential for STT, since the bladder wall must be lit from inside the bladder. Those two advantages permit complete dissection of the affected area. From that perspective, the laparoscopic procedure in the present study was reasonable. The third advantage is avoiding excessive resection. Since endometriosis originates outside the bladder and invades toward the urothelial mucosa [11], compared with affected abdominal wall, affected bladder wall is often small. Dissecting only from abdominal side by laparoscopy causes excessive resection of bladder wall and urothelial mucosa. To avoid excessive resection, deciding incision line and dissecting from outside and inside of the bladder simultaneously is necessary. Adding TU resectoscope to LPC may have merit in avoiding excessive resection of the bladder wall and urothelial mucosa to maintain postoperative bladder function.

In the present laparoscopic surgery, STT was performed in all cases. With STT, the edge of the affected area and the true incision line could be identified accurately and precisely. Seracchioli et al. [8] reported the light-to-light technique of LPC for BE, which used light-on laparoscopy and light-on cystoscopy during the procedure. The aim of light-to-light technique is to preserve urothelial mucosa and maintain bladder function. We discovered that we could identify the edge of the affected area precisely and easily by turning off the laparoscopic light source. Due to the difference in wall thickness, normal bladder wall is easy to distinguish from affected wall. Furthermore, $\mathrm{BE}$ is a rare disease. Urologists usually have little experience treating $\mathrm{BE}$, and it is too difficult for them to distinguish affected wall from normal wall. However, the surgical procedure itself is simple. Even the urologist with no experience treating BE can easily complete this procedure. However, the most important factor for curative surgery is to identify the edge of the affected area precisely and dissect it completely. STT makes that process simpler.

To ensure complete cure for a rare disease, this simple and novel procedure is thought to be reasonable. It resulted in no perioperative adverse events such as hydronephrosis or pyelonephritis, and the STT operative time including TU resection time was as long as other reports of simple LPC. During the average follow-up of 20.4 months, there was no recurrence, and two patients became pregnant.

In the present study, the difference in optical transparency between BE-affected bladder wall and normal bladder wall was demonstrated. By monitoring with light-off 
laparoscopy, this difference can be observed more easily and clearly. Lee et al. [14] reported "endoscopic submucosal cutting and light transmission" in laparoscopic gastric local resection. The basis of this procedure is similar to that of STT. They resected the gastric submucosa on the incision line by endoscopic resection first to create the difference in wall thickness. They subsequently monitored the lesion by laparoscopy after turning off the laparoscopic light source. They also reported that laparoscopic local gastric dissection could be performed precisely and safely using this procedure.

Endometriotic cells might be planted during past surgery. This iatrogenic route was said to be one of the pathogenetic mechanisms of BE [15]. Donnez et al. [16] reported that approximately $50 \%$ of patients with endometriosis of the bladder or ureter had undergone previous pelvic surgeries. In the present study, there were only 3 patients (25\%) with a past history of pelvic surgery.

The limitations of this study include the small sample size, its retrospective design and follow-up schedule. Thus, a prospective, randomized study with a larger number of patients and longer-term follow-up is necessary to obtain further evidence.

In the present study, LPC was performed with a TU resectoscope for $\mathrm{BE}$, achieving complete cure in all patients. With STT, the edge of the affected area and the true incision line could be correctly identified. Our good results are likely associated with LPC with the TU resectoscope and STT as surgical management for BE. Although only limited evidence currently supports the use of STT, further studies to obtain additional data may support its use to obtain better outcomes in cases of BE.

\section{Conclusion}

LPC was performed with a TU resectoscope for BE, achieving complete cure in all patients. With STT, the edge of the affected area and the true incision line can be accurately identified. During the average follow-up of 20.4 months, there was no recurrence, and two patients became pregnant.

\section{Statement of Ethics}

This study protocol was approved by the committee on human research of Nippon Medical School. Informed consent was obtained from the study participants, including consent to participate and to publish the findings.

\section{Disclosure Statement}

We have no conflict of interest to declare.

\section{Funding Sources}

None.

\section{References}

1 Pérez-Utrilla Pérez M, Aguilera Bazán A, Alonso Dorrego JM, Hernández A, de Francisco MG, Martín Hernández M, et al. Urinary tract endometriosis: clinical, diagnostic, and therapeutic aspects. Urology. 2009 Jan; 73(1):47-51.

2 Chapron C, Fauconnier A, Vieira M, Barakat $H$, Dousset B, Pansini V, et al. Anatomical distribution of deeply infiltrating endometriosis: surgical implications and proposition for a classification. Hum Reprod. 2003 Jan;18(1): 157-61.

3 Leonhartsberger N, Zelger B, Rehder P. Intrinsic endometriosis of ureter and bladder in young women without gynecological symptoms. Urol Int. 2008;80(2):222-4.

4 Villa G, Mabrouk M, Guerrini M, Mignemi G, Montanari G, Fabbri E, et al. Relationship between site and size of bladder endometriotic nodules and severity of dysuria. J Minim Invasive Gynecol. 2007 Sep-Oct;14(5):628-32.

5 Fauconnier A, Chapron C, Dubuisson JB, Vieira M, Dousset B, Bréart G. Relation between pain symptoms and the anatomic loca- tion of deep infiltrating endometriosis. Fertil Steril. 2002 Oct; 78(4):719-26.

6 Manganaro L, Fierro F, Tomei A, Irimia D, Lodise P, Sergi ME, et al. Feasibility of 3.0T pelvic MR imaging in the evaluation of endometriosis. Eur J Radiol. 2012 Jun;81(6):13817.

7 Bulun SE. Endometriosis. N Engl J Med. 2009 Jan;360(3):268-79.

8 Seracchioli R, Mannini D, Colombo FM, Vianello F, Reggiani A, Venturoli S. Cystoscopy-assisted laparoscopic resection of extramucosal bladder endometriosis. J Endourol. 2002 Nov;16(9):663-6.

9 Garry R. Endometrial ablation and resection: validation of a new surgical concept. $\mathrm{Br} \mathrm{J} \mathrm{Ob-}$ stet Gynaecol. 1997 Dec;104(12):1329-31.

10 Maccagnano C, Pellucchi F, Rocchini L, Ghezzi M, Scattoni V, Montorsi F, et al. Diagnosis and treatment of bladder endometriosis: state of the art. Urol Int. 2012;89(3):249-58.

11 Kołodziej A, Krajewski W, Dołowy Ł, Hirnle L. Urinary tract endometriosis. Urol J. 2015 Sep;12(4):2213-7.
12 Nezhat CH, Malik S, Osias J, Nezhat F, Nezhat C. Laparoscopic management of 15 patients with infiltrating endometriosis of the bladder and a case of primary intravesical endometrioid adenosarcoma. Fertil Steril. 2002 Oct; 78(4):872-5.

13 Chapron C, Dubuisson JB. Laparoscopic management of bladder endometriosis. Acta Obstet Gynecol Scand. 1999 Nov;78(10):887-90.

14 Lee YJ, Park JH, Jeong SH, Ha CY, Kwag SJ, Kim JY, et al. A noble method for intraoperative fine localization during laparoscopic gastric local resection: endoscopic submucosal cutting and light transmission. Surg Endosc. 2015 Aug;29(8):2456-61.

15 Somigliana E, Vercellini P, Gattei U, Chopin N, Chiodo I, Chapron C. Bladder endometriosis: getting closer and closer to the unifying metastatic hypothesis. Fertil Steril. 2007 Jun; 87(6):1287-90

16 Donnez J, Van Langendonckt A, CasanasRoux F, et al. Current thinking on the pathogenesis of endometriosis. Gynecol Obstet Invest. 2002;54 Suppl 1:52-8. 